\title{
Vitamina D y piel
}

\author{
Vitamin D and skin
}

\section{Ana Lucía Molina', Ángela Londoño²}

1. Médica, residente III de Dermatología, Facultad de Medicina, Escuela de Ciencias de la Salud, Universidad Pontificia Bolivariana, Medellín, Colombia

2. Médica dermatóloga y epidemióloga; docente, Facultad de Medicina, Escuela de Ciencias de la Salud, Universidad Pontificia Bolivariana, Medellín, Colombia

\section{Resumen}

La vitamina D es un nutriente esencial que puede obtenerse por exposición solar, suplementos o en la dieta. Históricamente, se ha considerado como fundamental en la homeostasis del calcio y el fósforo, pero actualmente se han encontrado múltiples y diferentes funciones en la regulación del crecimiento y proliferación celular, como inmunomodulador, como antioxidante, en el control del sistema renina-angiotensina-aldosterona y en la producción de insulina, entre otras. Sus efectos están regulados por factores endógenos y exógenos, y su deficiencia se ha asociado a diferentes enfermedades como cáncer, enfermedades autoinmunitarias, enfermedades cardiovasculares e infecciones.

El objetivo de este artículo fue hacer una revisión actualizada y completa sobre la vitamina D, sus efectos, sus funciones y las posibles complicaciones de su deficiencia. Se presenta esta revisión por ser un tema de actualidad, de gran controversia e importancia en el campo de la Dermatología.

Palabras Clave: vitamina D, neoplasias cutáneas, radiación ultravioleta.

\section{Summary}

Vitamin D is an essential nutrient, which may be obtained through sunlight exposition, supplements or healthy diet. It has been considered fundamental in calcium and phosphorus balance, but lately many other different functions have been published such as growth and cellular proliferation, immune function, control of insulin production, and rennin and aldosterone function, among others. The effects of vitamin D are regulated by endogenous and exogenous factors and its deficiency has been related to different pathologies such cancer, auto-immune and cardiovascular diseases and infections.

The purpose of this article was to make an updated review of vitamin D, its effects, functions and the possible complications of its deficiencies. We present this review because it is a current subject and very controversial and important in dermatological practice.

KEYWORDS: Vitamin D, skin cancer, solar UV radiation

\section{Correspondencia:}

Ana Lucía Molina

Email:

anamolina2@une.net.co

Recibido: 18 de junio de 2012. Aceptado: 25 de agosto de 2012.

No se reportan conflictos de intereses.

\section{Introducción}

La vitamina $\mathrm{D}$ es un nutriente esencial que puede obtenerse por la radiación solar, en la dieta o por suplementos $^{1}$. Una de las funciones más antiguas y reconocidas de la vitamina D es mantener la hemostasia del calcio y del fósforo. Actualmente, se considera más como una hormona que como una vitamina. Muchos autores se refieren a ella como la hormona de la luz solar, ya que se ha podido demostrar su papel como regulador de funciones de sistemas genómicos complejos, como son la diferenciación y el crecimiento celular, la regulación inmunitaria y la transformación neoplásica. Por todo esto, los niveles de vitamina D se deben mantener 


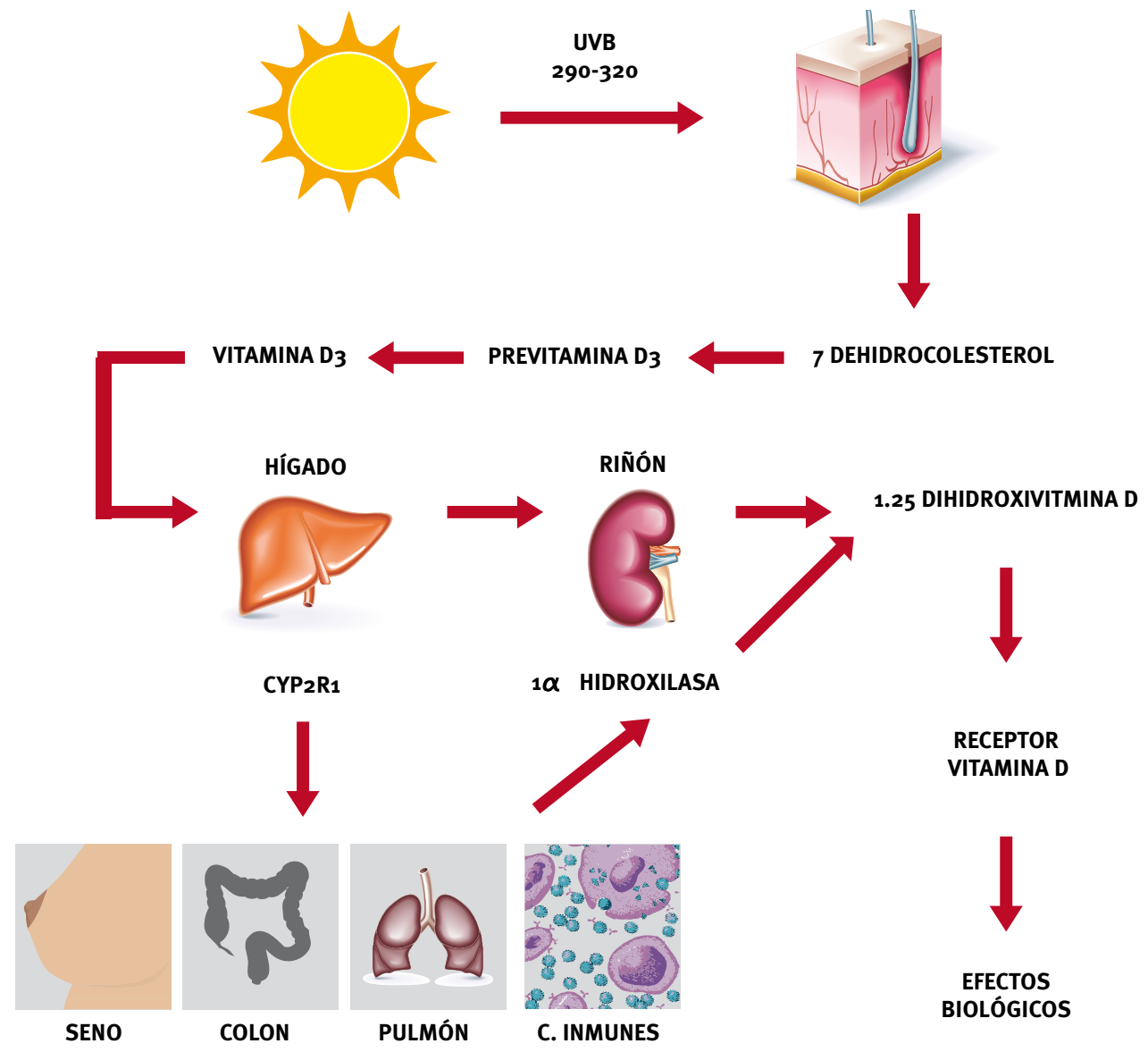

Figura 1. Fisiología de la vitamina $D$

en un rango adecuado para evitar un impacto negativo en la salud, además de los efectos ya conocidos como son el raquitismo y la osteomalacia. Recientemente, se ha asociado con el desarrollo de múltiples neoplasias, enfermedades autoinmunitarias, infecciones y enfermedades cardiovasculares, entre otras ${ }^{2,3}$.

Para los humanos, la historia de la deficiencia de la vitamina D y sus consecuencias en la salud se apreciaron por primera vez en las zonas industrializadas del norte de Europa, donde existía gran polución y no había suficiente exposición solar de los niños, lo cual resultó en una enfermedad deformante de los huesos que fue reconocida por Glisson, en 1651, como raquitismo. En el siglo XX, 80 a $90 \%$ de los niños que vivían en Holanda y en Boston sufrían de raquitismo. En 1919, Huldschinski, reportó que los niños que sufrían raquitismo y eran expuestos a las lámparas de mercurio presentaban mejoría en las radiografias óseas. En 1921, Hess y Unger descubrieron que los niños que sufrían de raquitismo y se exponían a la luz solar presentaban mejoría notable de sus lesiones. Esto llevó al gobierno de los Estados Unidos a recomendar a todos sus ciudadanos a exponer a sus hijos al sol para prevenir el raquitismo. Steenbock hizo un estudio en el cual se demostró la importancia de fortificar la leche con vitamina D y esto llevó a que se erradicara el raquitismo en Estados Unidos y en Europa ${ }^{1,4,5}$.

\section{Fisiología}

Como se mencionó anteriormente, la vitamina D puede obtenerse de la dieta, en suplementos o por la acción de la luz solar en la piel, principal fuente de vitamina D, que contribuye el 90 \% (Figura 1). La exposición de la piel a los rayos ultravioleta de tipo B (UVB), con una longitud de onda de 290 a $315 \mathrm{~nm}$, induce la conversión fotolítica del 7-dehidrocolesterol a previtamina D3. Esto ocurre por unión de los carbones 9 y 10. La previtamina D3 es convertida rápidamente a vitamina $\mathrm{D}_{3}$, conocida también como colecalciferol, por un proceso depen- 


\section{"Para los humanos, la historia de la deficiencia de la vitamina D y sus consecuencias en la salud se apreciaron por primera vez en las zonas industrializadas del norte de Europa, donde existía gran polución y no había suficiente exposición solar de los niños, lo cual resultó en una enfermedad deformante de los huesos que fue reconocida por Glisson, en 1651, como raquitismo".}

diente del calor ch, $^{2,6}$. Aproximadamente, $65 \%$ del 7-dehidrocolesterol se encuentra en la epidermis, el resto se encuentra en la dermis. En algunos estudios experimentales se ha demostrado que el $50 \%$ de la previtamina D3 se puede convertir en la piel a vitamina D3 en 2,5 horas y, 12 a 24 horas luego de la exposición a los rayos UVB, las concentraciones de vitamina $\mathrm{D} 3$ en circulación son las máximas $4,6,7,8$.

Es importante saber que la exposición solar excesiva no produce exceso de vitamina D3 o previtamina $D_{3}$, porque cualquier exceso de estas es destruido por la luz solar. Posteriormente, la vitamina D3 pasa al hígado donde ocurre el primer paso en la activación metabólica de la vitamina $\mathrm{D}$, el cual consiste en la hidroxilación del carbón 25 por enzimas del citocromo p 450, principalmente la CYP2R1, y esto lleva a la producción de 25-hidroxivitamina D (25-OH vitamina D), la cual se considera como el principal indicador del estatus de vitamina $\mathrm{D}$ en cada paciente; este se utiliza porque se puede medir fácilmente, tiene la vida media más prolongada en la circulación ( 2 a 3 semanas) y se correlaciona adecuadamente con el cuadro clínico de los pacientes ${ }^{5,9}$.

El segundo paso en la activación de la vitamina D es la formación de 1.25 dihidroxivitamina $\mathrm{D}$, la forma activa de la vitamina $\mathrm{D}$, conocida también como calcitriol, a partir de la 25-OH vitamina $\mathrm{D}$, lo cual ocurre en el riñón, principalmente; esto se lleva a cabo por una enzima llamada 25-hidroxiviamina D-1 $\alpha$-hidroxilasa (CYP27B1).

La producción de esta forma activa es regulada por la acción de la paratohormona (PTH), los niveles séricos de calcio y fósforo y el factor de crecimiento de los fibroblastos. La 1- $\alpha$-hidroxilasa se ha encontrado en muchos sitios diferentes al riñón, como son: próstata, seno, colon, pulmones, células pancreáticas, monocitos, células paratiroideas, queratinocitos, melanocitos y sebocitos. En estos tejidos, la enzima actúa de forma paracrina y actúa en células vecinas pero no produce aumento del calcitriol en el plasma ${ }^{3,10,11}$.

La 1- $\alpha$-hidroxilasa también es controlada por el calcitriol, el cual hace una retroalimentación negativa sobre esta para evitar la intoxicación por vitamina $\mathrm{D}$.

El calcitriol es controlado e inhibido por una enzima llamada 24-hidroxilasa vitamina $\mathrm{D}$, que lo convierte en un metabolito inactivo que es excretado por la bilis. Los metabolitos de la vitamina $\mathrm{D}$ son lipofílicos y deben ser trasportados a la circulación unidos a proteínas; la más importante es la proteína unidora de vitamina D (Vitamin D Binding Protein, DBP), pero también, puede estar unida a albúmina y lipoproteínas. El nivel de DBP se disminuye cuando existe enfermedad hepática, síndrome nefrótico o desnutrición, y se encuentra aumentada en el embarazo y durante el tratamiento con estrógenos.

La mayoría de las acciones del calcitriol requieren de un receptor de gran afinidad, el cual es llamado receptor de alta afinidad de la vitamina D (Vitamin $D R e$ ceptor, VDR). Este actúa como un factor de transcripción ligando activado; el dominio ligando localizado en la porción $\mathrm{COOH}$ terminal del VDR es el responsable de la gran afinidad del calcitriol.

La porción responsable del calcitriol de la unión al receptor es el anillo A que contiene el grupo 1- $\alpha$-hidroxil. El VDR se encuentra en múltiples sitios diferentes al riñón, como son: queratinocitos, osteoblastos, linfocitos $\mathrm{T}$ y $\mathrm{B}$, intestino delgado, próstata, colon, seno, corazón, cerebro, gónadas, hueso, folículo piloso, ovario, placenta y útero. Cuando el calcitriol se une al receptor en los diferentes órganos, se produce la respuesta biológica esperada ${ }^{7,12,13}$.

La segunda forma más importante para obtener la vitamina D, es a partir de la dieta y los suplementos vitamínicos. Pocos alimentos contienen vitamina D3 y D2; estas se absorben en el sistema gastrointestinal y luego 


\begin{tabular}{|cc}
\hline $\begin{array}{c}\text { Órgano } \\
\text { Hueso, músculo } \\
\text { Células inmunes }\end{array}$ & $\begin{array}{c}\text { Función } \\
\text { SRAA }\end{array}$ \\
\hline Inducción péptidos antimicrobianos, regulación CPA, células Ty del IFN $\gamma$ \\
\hline Pancreas & Regulación de presión arterial, electrolitos y hemostasis \\
\hline SNC & Regula producción insulina \\
\hline Diferentes células & Neuro-protección \\
\hline
\end{tabular}

Figura 2. Funciones de la vitamina D.

son incorporadas a quilomicrones y transportadas al sistema linfático. Finalmente, pasan a la circulación venosa y sufren el mismo proceso fisiológico que la vitamina $\mathrm{D}$ producida por la luz solar para, después, convertirse en calcitriol. El aceite de pescado, principalmente de salmón, es una fuente excelente de vitamina D y contiene 500 a 1.000 UI por 3,5 onzas. Las comidas fortificadas con vitamina D incluyen la leche, el jugo de naranja, el pan, las margarinas, el queso, el yogur y los cereales, pero en Europa solo está fortificada la leche y prohiben la fortificación de otros alimentos por el riesgo de hipercalcemia. Cuando algunas circunstancias sociales, ambientales o fisiológicas previenen una adecuada exposición solar, debe ocurrir compensación del consumo de vitamina $\mathrm{D}$ en la dieta para prevenir su deficiencia y sus consecuencias ${ }^{14,15,16}$.

\section{Funciones}

El calcitriol tiene funciones biológicas clásicas y no clásicas. Las clásicas son las funciones genómicas que se llevan a cabo mediante la regulación en la transcripción de genes y el resultado final es mantener la homeostasis del calcio por su acción en los riñones, en el intestino, el hueso y la paratohormona (FIgURA 2). El calcitriol producido en los riñones induce la absorción intestinal de calcio, control la remodelación del hueso e inhibe la función de la paratohormona. En el intestino aumenta la eficacia para absorber el calcio y el fósforo, por la interacción del calcitriol con el complejo del receptor formado por VDR y el receptor del ácido retinoico X, y este es llevado a la circulación general mediante la bomba $\mathrm{Na}-\mathrm{Ca}^{++}$-ATPasa. Luego es reconocido y captado por los osteoblastos, lo cual aumenta la expresión del receptor activador nuclear del factor kappa $\beta$ (RANKL) en los preosteoclastos, lo que induce su maduración hacia osteoclastos. Los osteoclastos maduros eliminan el calcio y el fósforo del hueso, y así, se mantienen sus niveles normales en la sangre. En el esqueleto, la vitamina D es esencial para el desarrollo y el mantenimiento de la mineralización ósea ${ }^{8,17,18}$.

Las funciones no clásicas de la vitamina D se llevan a cabo mediante respuestas no genómicas, lo cual ocurre por activación de múltiples señales de transcripción; su efecto se produce por la interacción del calcitriol con el VDR en las diferentes células y tejidos en los cuales se encuentra. Las principales funciones no clásicas son las que se exponen a continuación ${ }^{1,2,19-21}$.

\section{DIFERENCIACIÓN Y PROLIFERACIÓN CELULAR}

Como ya se mencionó, múltiples células tienen VDR, entre ellas, los queratinocitos. Se ha encontrado que la interacción entre el calcitriol y el VDR en estas células regula múltiples genes que son importantes en la diferenciación y crecimiento celular, en la inflamación y en la cicatrización. Esta interacción regula la proliferación epidérmica en la capa basal y promueve la diferenciación secuencial de los queratinocitos. La pérdida del VDR produce alteración en la diferenciación epidérmica, lo que resulta en aumneto de la proliferación de las capas basales. Se ha encontrado que las concentraciones bajas de calcitriol producen estimulación del crecimiento de los queratinocitos in vitro, mientras que sus altas concentraciones producen inhibición del crecimiento. Esto se ha relacionado con el efecto que tiene el calcitriol en el aumento de los ligandos promotores del crecimiento; por esta razón, dosis altas de calcitriol o análogos de la vitamina $\mathrm{D}$ son buenas alternativas en el tratamiento de enfermedades cutáneas hiperproliferativas, como la psoriasis ${ }^{21-24}$.

El calcitriol induce la producción de esfingomielinasa, la cual convierte la esfingomielina en ceramidas. Las 
ceramidas estimulan el efecto prodiferenciador de los queratinocitos; además, este juega un papel muy importante en la inducción de apoptosis en diferentes células, incluyendo los queratinocitos. En concentraciones fisiológicas, el calcitriol no inicia la apoptosis en queratinocitos cultivados, pero, por el contrario, causa resistencia contra la acción proapotótica de las ceramidas, radiación de los rayos UV y FNT $\alpha$, y aumenta la filagrina y las glucoceramidas, que son importantes en el mantenimiento de la integridad de la epidermis s $^{25,26}$.

Recientemente, se ha demostrado que el calcitriol es importante en el control de múltiples tumores, porque induce la transcripción de algunos genes que son capaces de detener el crecimiento del linaje de macrófagos $\mathrm{y}$ monocitos, $\mathrm{y}$ favorece su diferenciación; induce una proteína supresora de oncogenes llamada C/EBP, que inhibe la ciclina d1, implicada en tumores epiteliales; reduce los niveles de HRPA20, una fosfoproteína que aumenta el crecimiento y la supervivencia de células dependientes de prolactina -importante en el cáncer de mama-; al interactuar con los VDR en las células de la próstata, promueve el factor de crecimiento insulinalike binding protein 3 y genes receptores de insulina, lo cual juega un papel fundamental en el crecimiento y en el funcionamiento normal de la próstata y en la carcinogénesis prostática; $y$, finalmente, en las células de cáncer de mama, el calcitriol induce apoptosis mediante la modulación del bcl2 y bax, y, también, aumenta el calcio intracelular que activa la proteasa proapoptótica dependiente de calcio. Estos hallazgos demuestran que los efectos proapotóticos y antiaproptóticos son importantes en el desarrollo y la función del tejido normal, y en la inducción de detención del crecimiento del cáncer y alteraciones no malignas de hiperproliferación ${ }^{26,27}$.

\section{INMUNOMODULACIÓN}

Los efectos inmunomoduladores de la vitamina D fueron descubiertos por la frecuente asociación de infecciones recurrentes en pacientes con raquitismo 28 . Como se sabe, varias células que están involucradas en las reacciones inmunológicas tienen la maquinaria necesaria para la producción de vitamina $\mathrm{D}$, como son los monocitos, los linfocitos $\mathrm{T}$ y $\mathrm{B}$ y las células de Langerhans. El calcitriol induce la producción de C/ EBP y p21, los cuales median la función inmunitaria de monocitos-macrófagos; el C/EBP es crítico para la producción de IL12 (media la acción TH1) y para la acción contra virus y bacterias y la antitumoral de los macrófagos. La 1- $\alpha$-dihidroxivitamina $\mathrm{D}$ inhibe la activación de las células $\mathrm{T}$ e induce la generación de linfocitos $\mathrm{T}$ reguladores $\mathrm{CD}_{25}+/ \mathrm{CD} 4+$. En las células dendríticas, el calcitriol inhibe la maduración e induce un fenotipo que promueve la tolerancia e inhibe la inmunidad luego de la estimulación antigénica; también, en estas suprime la expresión de moléculas del complejo mayor de histocompatibilidad de tipo II y moléculas coestimuladora, como CD40, CD80, y CD86; estimula la producción de IL10 e inhibe la producción de IL12, lo cual lleva a una supresión en la activación de células T.

Actualmente, se está considerando una relación entre la vitamina D y la dermatitis atópica. Se ha encontrado en algunos estudios que los pacientes con dermatitis atópica tienen niveles bajos de de vitamina D en comparación con los controles; también, se ha demostrado que la vitamina $\mathrm{D}$ inhibe in vitro la IgE y la reacción cutánea mediada por esta. Estos efectos inmunomoduladores hacen que los análogos de la vitamina D se conviertan en buenas opciones de tratamiento en enfermedades inflamatorias, como la dermatitis atópica y la dermatitis de contacto ${ }^{25,26,29-31}$.

Es importante reconocer el papel de la vitamina D en la infección por Mycobacterium tuberculosis, que puede explicarse por la producción local de vitamina D en los macrófagos activados. En estudios recientes se han dado nuevos datos sobre la regulación en la producción de citocinas por los macrófagos mediados por la vitamina D. El interferón $\gamma$ (IF $\gamma)$, la citocina que se encuentra elevada en la infección por micobacterias, es un inductor poderoso de la expresión del gen de la 1- $\alpha$-hidroxilasa en los macrófagos y, por lo tanto, es importante en la producción de calcitriol. Cuando el IF $\gamma$ está aumentado, no ocurre catabolismo del calcitriol y, por el contrario, se aumenta. El calcitriol producido por los macrófagos induce la función antibacteriana de los macrófagos y aumenta también la producción y actividad del IF $\gamma{ }^{32}$. Cuando existe un daño en los mecanismos de barrera, los patógenos son reconocidos por los receptores toll-like y existe una regulación positiva de la enzima $\mathrm{CYP}_{27} \mathrm{~B} 1$; hay un aumento de calcitriol y este actúa en los VDR de linfocitos $\mathrm{T}$ y $\mathrm{B}$, monocitos y células asesinas naturales, y se induce la producción de catelicidinas, defensinas y péptidos antimicrobianos ${ }^{33,34}$, los cuales pueden estimular el sistema inmunitario adaptativo e innato. El efecto antimicrobiano de las catelicidinas se ha demostrado contra M. tuberculosis. Liu, et al., demostraron que la adición de calcitriol a macrófagos infectados por esta bacteria, llevaba a una disminución en el número de bacilos y que la inducción de catalicidina producía autofagia, que significa la ingestión de material secuestrado dentro de los fagosomas ${ }^{35}$.

Ho-Pham, et al., evaluaron la asociación entre el estatus de la vitamina $\mathrm{D}$ y de la paratohormona, y el riesgo de desarrollar tuberculosis en una población vietnamita. Se incluyeron 166 pacientes con tuberculosis y 


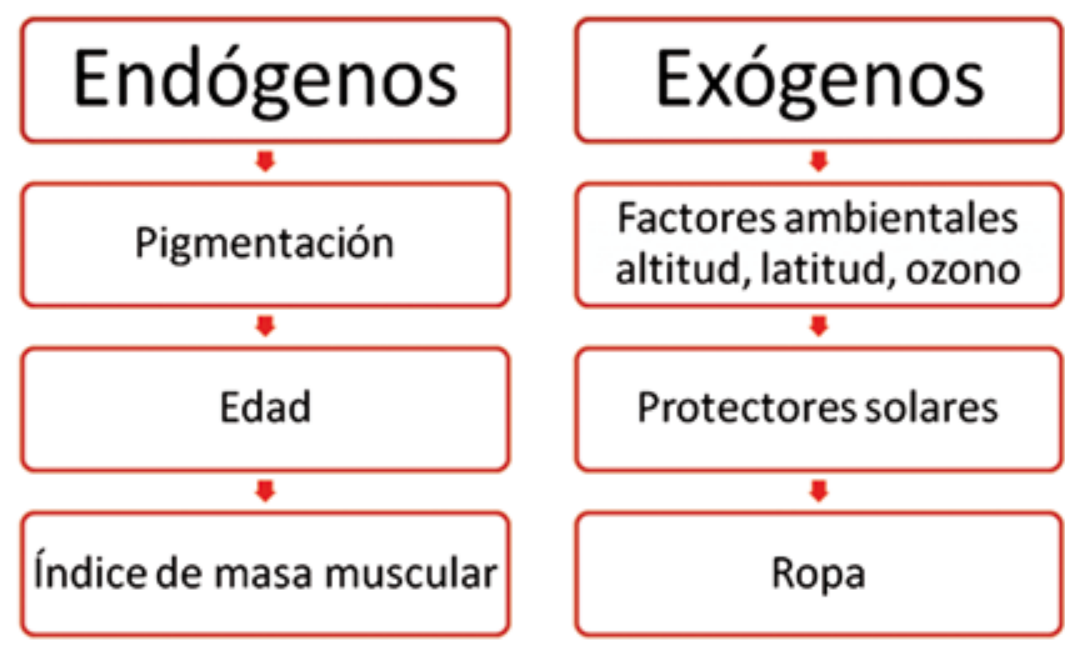

Figura 3. Factores que afectan la producción de vitamina $\mathrm{D}$
219 controles. Encontraron que la prevalencia de insuficiencia de vitamina $\mathrm{D}$ fue de $34,4 \%$ en los hombres con tuberculosis y de 19,5\% en los controles ( $p=0,001)$; no obstante, en las mujeres no hubo una diferencia estadísticamente significativa. Estos resultados sugieren que la insuficiencia de vitamina $\mathrm{D}$ es un factor de riesgo para la tuberculosis en hombres pero no en mujeres, pero se requieren más estudios para confirmar este hallazgo ${ }^{36}$.

\section{ANTIOXIDANTE}

Los rayos UVB son el principal factor de riesgo para el desarrollo de cáncer no melanoma; estos inducen la producción de dímeros de ciclobutano de pirimidina (CPD) y otros foto-productos que inducen apoptosis e IL6. El cáncer ocurre por mutaciones desarrolladas por CPD no corregidas por el ADN. Recientemente, se ha demostrado que el calcitriol induce protección en los queratinocitos contra los CPD y parece tener un efecto fotoprotector en los queratinocitos in vitro, porque induce la producción de la proteína metalotioneína, la cual es reconocida como antioxidante $23,37,38$.

\section{SISTEMA RENINA-ANGIOTENSINA- ALDOSTERONA}

Este sistema juega un papel fundamental en la regulación de la presión arterial, los electrolitos y la homeostasis. El calcitriol actúa como regulador negativo en el sistema renina-angiotensina-aldosterona. En ratones sin VDR se han demostrado niveles altos de renina y angiotensina, lo cual se asocia a hipertensión, cardiomiopatía hipertrófica y aumento en la ingestión de agua.
Los VDR se han encontrado en músculo liso, endotelio y cardiomiocitos, y se ha demostrado que el calcitriol regula la proliferación y crecimiento de las células del músculo liso vascular y los cardiomiocitos.

\section{SECRECIÓN DE INSULINA}

En animales experimentales, la deficiencia de vitamina D se asocia a diabetes más agresiva y de inicio más temprano; probablemente, esto esté relacionado con defectos en la función inmunitaria y alteración en la secreción de insulina mediada por glucosa, y puede ser reversado con la administración de calcitriol. Mediante el VDR, el calcitriol controla el influjo de calcio a los islotes pancreáticos, lo cual afecta la liberación de insulina. El hallazgo de la enzima 1- $\alpha$-hidroxilasa en las células pancreáticas, ha llevado a pensar que existe una función autocrina mediada por el calcitriol sobre el control de la insulina.

\section{CONTROL DEL SISTEMA NERVIOSO CENTRAL}

Existe evidencia reciente que sustenta el papel de la vitamina D en la función y el desarrollo cerebrales, incluyendo neuroprotección in vitro; esto se sucede mediante inmunomodulación, regulación del calcio neuronal, mecanismos antioxidantes, aumento en la conducción nerviosa y por mecanismos de desintoxicación. En pacientes con Alzheimer, se encontraron puntajes más altos del cuando existían niveles suficientes de vitamina D, en comparación con cuando son insuficientes ${ }^{1,39,40}$. 


\section{Parámetros}

Aunque no existe un consenso sobre cuáles son los niveles óptimos de vitamina $\mathrm{D}$, se considera que son deficientes cuando son menores de $20 \mathrm{ng} / \mathrm{ml}$, insuficientes, cuando están entre 21 y $29 \mathrm{ng} / \mathrm{ml}$, y suficientes, cuando están por encima de $30 \mathrm{ng} / \mathrm{ml}$; la intoxicación por vitamina D se puede producir con niveles por encima de $150 \mathrm{ng} / \mathrm{ml}$. Con estos parámetros, se ha estimado que, aproximadamente, un billón $\left(10^{9}\right)$ de personas en todo el mundo tiene deficiencia o insuficiencia de vitamina D. Estudios recientes demuestran que, aproximadamente, 40 a $100 \%$ de los ancianos de ambos sexos en Estados Unidos y Europa, más del $50 \%$ de las mujeres posmenopáusicas que toman suplemento de calcio para osteoporosis, tienen niveles subóptimos de esta vitamina ${ }^{41}$. Los niños y adolescentes también están en riesgo de sufrir deficiencia; en un estudio en Boston, se encontró que $52 \%$ de preadolescentes hispanos y negros, y $40 \%$ de los blancos tenían niveles de vitamina D menor de $20 \mathrm{ng} / \mathrm{ml}$. En otro estudio se encontró que $42 \%$ de las mujeres de raza negra de 15 a 49 años de edad tenían niveles menores de $25 \mathrm{ng} / \mathrm{ml}$ en Estados Unidos y $32 \%$ de los médicos residentes sanos tenía niveles de menos de $20 \mathrm{ng} / \mathrm{ml}$, a pesar de tomar un vaso de leche al día, tomar multivitamínicos y comer salmón una vez por semana.

Quienes viven cerca a la línea del Ecuador y se exponen al sol sin protección, tienen niveles adecuados (mayores de $30 \mathrm{ng} / \mathrm{ml}$ ), pero, inclusive en verano, si se cubren del sol es común la deficiencia ${ }^{12}$. A pesar de que la mayoría considera los niveles mayores de $30 \mathrm{ng} / \mathrm{ml}$ adecuados, muchos creen que esto no es suficiente y que se necesitan niveles de 50 a $80 \mathrm{ng} / \mathrm{ml}$ para lograr la prevención de enfermedades crónicas y cáncer ${ }^{22-45}$.

\section{Factores que afectan la producción}

Es importante reconocer todos los factores que afectan la producción de vitamina D. Estos se pueden clasificar en endógenos y exógenos (Figura 3).

\section{FACTORES ENDÓGENOS}

\section{Pigmentación}

La melanina es un protector solar natural excelente, que absorbe eficientemente la radiación ultravioleta, incluyendo los rayos UVB, por lo cual compite por los fotones de los rayos UVB y, por lo tanto, disminuye la eficacia de la fotosíntesis del precolecalciferol. En un estudio en 182 personas, quisieron evaluar la importancia del nivel previo de vitamina $\mathrm{D}$, del color de la piel y del nivel del colesterol qué tanto afecta la producción de vitamina $\mathrm{D}$, y encontraron que el nivel basal de vitamina es importante en su producción después de la exposición solar; aquellos pacientes que tenían niveles de vitamina D insuficientes, respondían mejor a la exposición a la UVB. Se desconoce la causa de esto, pero se puede relacionar con que la $25-\mathrm{OH}$ vitamina D inhibe la 25-hidroxilasa en el hígado; el hígado promueve la hidroxilación de vitamina D3 hacia 25-hidroxivitamina D. También, se encontró que el nivel de colesterol era importante. Sostienen que un nivel bajo de colesterol puede ser problemático para la síntesis de vitamina $\mathrm{D}$ cuando su fuente es la comida; la síntesis se inicia en el epitelio intestinal con la oxidación del colesterol de la comida o de la bilis hacia 7-dehidrocolesterol, el cual es transportado hacia la piel (epidermis) donde ocurre el mismo proceso con los rayos UVB. No obstante, hasta el momento no se ha visto que el tratamiento con estatinas afecte la producción de vitamina D. También, se concluyó que el color de la piel no importaba en la producción de vitamina D durante el invierno; puede que en verano sí sea diferente, pues se encuentra más melanina en las capas basales que puede competir con la producción de vitamina $\mathrm{D}^{46}$.

En otro estudio se encontró que los pacientes de raza blanca y negra tienen la misma capacidad de producir vitamina D. La diferencia está en la cantidad de melanina. Los pacientes de ambas razas se expusieron a la misma cantidad de sol; en los de raza negra se detectó una cantidad mínima de vitamina D. En este estudio se demostró que la conversión epidérmica del 7-dihidrocolesterol a previtamina D en la piel de fototipo II es, aproximadamente, 5 a 10 veces más eficiente que en una piel de fototipo $\mathrm{V}^{47,48}$.

\section{Edad}

El envejecimiento tiene un efecto importante en la piel. La producción de la vitamina D se ve afectada, ya que el grosor de la piel disminuye linealmente con el aumento de la edad luego de los 20 años y la concentración de 7-dihidrocolesterol en la epidermis a los 70 años es 20 $\%$ de la que encuentra a los 20 años. Se hizo un análisis comparando la concentración de vitamina $\mathrm{D}$ de un joven y un paciente de edad expuestos a la misma cantidad de sol, en el mismo lugar y por el mismo tiempo, y se demostró que en el joven la concentración máxima fue de $30 \mathrm{ng} / \mathrm{ml}$ y en adulto mayor (62 a 80 años) fue de $20 \mathrm{ng} / \mathrm{ml}$. Otros factores que ponen en riesgo a los pacientes de edad es la falta de movilidad, no exponerse 
al sol, no consumir suplementos, la disminución en la producción renal de calcitriol y la malabsorción ${ }^{47}$.

\section{Obesidad}

Se ha encontrado que los pacientes con peso corporal aumentado (\% de grasa) tienen niveles más bajos de vitamina $\mathrm{D}$, en comparación con personas con peso normal. Esto ocurre por baja disponibilidad de vitamina en la piel luego de la exposición solar, disminución en la capacidad de producción, aumento en el secuestro de la vitamina D en el tejido celular subcutáneo, deficiencia en la dieta y falta de exposición al sol. La evaluación de la concentración de vitamina D luego de 24 horas de irradiación de todo el cuerpo, era $57 \%$ más baja que en los controles no obesos ${ }^{49,50}$.

En un estudio en 127 niños, se evaluó la relación entre obesidad, deficiencia de vitamina $D$ y resistencia a la insulina, estación y raza. Se encontró que los pacientes obesos tenían concentraciones más bajas de vitamina $\mathrm{D}$, lo cual fue mucho mayor en otoño e invierno y en pacientes de raza negra e hispanos, y se halló una relación negativa entre los niveles de vitamina D y la hemoglobina $\mathrm{A}_{1} \mathrm{C}^{51}$.

\section{Otros factores}

Se ha encontrado que los pacientes quemados tienen niveles bajos de 7-dehidrocolesterol en la piel. Los pacientes con malabsorción, disminución en la producción (enfermedad hepática o renal), embarazo o lactancia, pueden estar en riesgo de desarrollar deficiencia de vitamina $\mathrm{D}^{47}$.

\section{FACTORES EXÓGENOS}

\section{Ozono}

El ángulo de cenit depende de la latitud, la estación y la hora del día. Mientras más directo sea el rayo solar (perpendicular), existe menor ángulo de cenit, es menor el recorrido de los rayos UV y es menor la absorción por la capa de ozono. La capa estratosférica absorbe en forma eficiente la radiación solar menor de $290 \mathrm{~nm}$. Los clorofluoro-hidrocarbonos producen disminución de esta capa, lo que resulta en transmisión de grandes cantidades de fotones ultravioletas de alta energía.

\section{Latitud}

El sol está presente siempre a latitudes altas y bajas. Los fotones de UVB disponibles para la producción de vitamina D solo están disponibles en una parte del día, por- que el sol en las latitudes altas no alcanza una posición tan alta en comparación con las latitudes bajas. Por lo tanto, los rayos UVB tienen una distancia más larga que recorrer y, cuando llegan a la superficie de la tierra, están más atenuados ${ }^{32}$. En regiones con latitudes altas, la sintesis de vitamina D durante el verano o la primavera parece ser suficiente para producir una buena reserva de vitamina $D$ en la grasa y el músculo para garantizar adecuados niveles en el invierno, pero, cuando se analizan los niveles de rayos UV, se demuestra que en una amplia zona en la región norte del hemisferio no hay suficiente rayos UV para permitir una producción adecuada de vitamina D, inclusive en pacientes con piel de fototipos I o II. En latitudes $40^{\circ} \mathrm{N}$ (Boston) existe radiación de UVB insuficiente para la producción de vitamina $\mathrm{D}$ desde noviembre hasta marzo; en Canadá, latitud $52^{\circ} \mathrm{N}$, la deficiencia va desde octubre hasta marzo ${ }^{48}$.

\section{Estación}

Es bien sabido que la deficiencia de vitamina D es más prevalente en los meses de invierno. En esta estación las personas pasan poco tiempo al aire libre y usan más ropa, lo cual disminuye el área de superficie expuesta a la luz solar. El ángulo de cenit del sol aumenta en el otoño y el invierno, y la cantidad de radiación solar que alcanza la tierra es mucho menor. En Boston se hizo un estudio para evaluar la estación y la hora del día en relación con las concentraciones de vitamina D y se encontró que en julio y junio, la fotólisis cutánea del 7-dehidrocolesterol a precolecalciferol estaba al máximo; de agosto a octubre, había una disminución gradual de la eficacia de conversión; en octubre, la conversión era menos de 4 $\%$ de 11:30 a.m. a 2:00 p.m., y de noviembre a marzo, no se detectaba o era mínima la conversión. En los Ángeles y Puerto Rico, la producción ocurre todo el año ${ }^{52}$.

\section{Altitud}

A mayor altitud existe menor distancia de los rayos solares y menor densidad de la capa de ozono, lo cual aumenta los rayos que atraviesan la atmósfera y llegan a la piel; por cada $300 \mathrm{~m}$ que aumente la altitud, se aumenta en $4 \%$ la cantidad de rayos que llegan a la piel.

\section{Ambiente}

En las ciudades industrializadas donde existe tanta contaminación ambiental, los rayos UVB no atraviesan la atmósfera y no son absorbidos por la piel, lo cual puede producir deficiencia de vitamina. La cobertura de nubes también es un factor importante; cuando están bajas y gruesas, tienen mayor habilidad de disminuir el paso de los rayos UVB; las nubes gruesas pueden prevenir que 
$99 \%$ de los rayos UVB alcancen la tierra y pueden prevenir la síntesis de vitamina ${ }^{47,52}$.

\section{Protectores solares}

La exposición solar produce efectos nocivos para la salud. Por esta razón, se adelantan campañas que recomiendan evitar la exposición solar y promueven el uso masivo de protectores solares. Este es un punto muy importante para los dermatólogos y, actualmente, existe controversia sobre su uso, ya que bloquean los mismos rayos UV que son necesarios para la producción de vitamina D. Uno de los primeros estudios fue el de Matsuoka, et al.; reclutaron 20 pacientes que se aplicaron un antisolar con ácido paraaminobezoico (PABA) antes de salir a la calle por más de un año. Se compararon con los de un grupo control que consistía en 20 pacientes blancos, sanos, con exposición solar similar. Se encontró que la concentración sérica de 25$\mathrm{OH}$ vitamina $\mathrm{D}$ era menor en el grupo de los casos que en sus controles, 9,1 $\pm 6,2$ Vs. 40,2 $\pm 3,2 \mathrm{nmol} / \mathrm{L}$, respectivamente, con una p significativa ${ }^{53}$.

Existen algunos grupos de pacientes en los cuales la exposición solar está contraindicada, ya sea porque exacerba su condición de base o por el alto riesgo de sufrir cáncer de piel, y son los que más utilizan los protectores solares; por lo tanto, se encuentran en riesgo de sufrir déficit de vitamina D. Algunos ejemplos son los pacientes con trasplantes, en tratamiento inmunosupresor, con lupus eritematosos sistémico y con algunos síndromes con predisposición a desarrollar prematuramente cáncer de piel (xerodema pigmentoso, síndrome de Gorlin, etc.), entre otros. Se han llevado a cabo múltiples estudios tratando de evaluar el riesgo de desarrollar déficit de vitamina $\mathrm{D}$, pero hasta el momento no existe un consenso. En un estudio que se hizo en 31 pacientes con trasplante renal, que utilizaban antisolar diariamente, ropa protectora y evitaban la exposición solar, se encontró que los niveles de $25-\mathrm{OH}$ vitamina D eran significativamente menores en los pacientes con trasplantes, en comparación con el grupo control ${ }^{54}$.

A pesar de que en muchos estudios se han encontrado niveles anormales de vitamina D por el uso de antisolares, hasta el momento existen pocos estudios a gran escala que pueda confirmar estos hallazgos. Marks, et al., hicieron un estudio doble ciego de asignación aleatoria controlado con placebo, en el cual se evaluaron los efectos del uso de protector solar de amplio espectro con factor de protección (FPS) 17, en pacientes sanos de Australia con niveles normales de vitamina D, y se compararon con un grupo que se aplicaba placebo. Se midió la vitamina $\mathrm{D}$ al inicio y al final, y se encontró que no hubo diferencia estadísticamente significativa en el aumento de $25-\mathrm{OH}$ vitamina $\mathrm{D}$ entre los dos grupos 55 .

La experiencia de la mayoría con el uso de protector solar es que, a pesar de su aplicación, se queman o broncean luego de la exposición casual al sol.

El factor de protección solar es estrictamente determinado por la Food and Drug Administration (FDA) con base en la aplicación de $2 \mathrm{mg} / \mathrm{cm}^{2}$, pero en un estudio se informó que la mayoría de la gente solo se aplica 0,5 $\mathrm{mg} / \mathrm{cm}^{2}$, lo cual reduce dicho factor de 16 a 2. Otros factores que influyen en que el protector solar no se utilice como se debe, que no se aplica sino hasta llegar a la playa, la uniformidad en la aplicación, la durabilidad, la cantidad, la aplicación periódica repetida y, finalmente, aunque se aplique correctamente, por definición no bloquea completamente los rayos UVB; un factor de protección solar de 15 permite la penetración de 1/15 o $6 \%$ de los fotones de UVB. Por todo lo anterior, es muy poco probable que el uso de protector solar produzca déficit de vitamina $\mathrm{D}^{56}$.

\section{Ropa}

Se ha demostrado que las personas que utilizan ropa de pies a cabeza por cuestiones culturales, pueden presentar déficit de vitamina $\mathrm{D}^{57}$.

\section{Consecuencias}

\section{ÓSEAS}

La consecuencia clínica establecida de la deficiencia de vitamina $D$ es el hiperparatiroidismo secundario, en el cual se compensa la inadecuada absorción del calcio intestinal con la movilización de calcio del hueso hacia el torrente sanguíneo y eliminando fósforo por la orina. Finalmente, el depósito inadecuado de calcio en el hueso lleva a una mineralización ósea inadecuada y esto produce raquitismo en niños y osteomalacia en adultos.

\section{CÁNCER}

La conexión entre cáncer y deficiencia de vitamina D se notó desde 1615, cuando se dieron cuenta de que los pacientes que se exponían más al sol tenían más riesgo de sufrir cáncer de piel, pero menor riesgo de otros tumores malignos ${ }^{58,59}$.

El déficit de vitamina $D$ se ha asociado al desarrollo de múltiples tumores malignos, los más frecuentes son de colon, de mama, de próstata y el melanoma.

El cáncer colorrectal es la tercera causa de cáncer en mujeres y hombres en Estados Unidos. En los estudios se ha demostrado una correlación inversa entre 


\section{"La deficiencia de vitamina $\mathrm{D}$ se ha asociado a diabetes mellitus de tipo 1, esclerosis múltiple, artritis reumatoide y lupus eritematoso sistémico, entre otras enfermedades".}

la exposición solar y el cáncer de colon. Grant, et al., demostraron que la radiación UVB se relacionaba inversamente con la mortalidad por diversos cánceres; la asociación más fuerte fue con cáncer colorrectal. También, se encontró que la mortalidad por cáncer de colon era 20 a $30 \%$ menos en los meses de otoño en comparación con los de invierno y que la exposición solar durante un periodo antes del diagnóstico se asociaba con mejor supervivencia porque los niveles de vitamina D estaban más altos ${ }^{60,61}$.

En un estudio de Wactawski-Wende, et al., doble ciego, de asignación aleatoria y controlado con placebo de 36.282 mujeres posmenopáusicas, 18.176 tomaban $1.000 \mathrm{mg}$ de calcio más 400 UI de vitamina D y 18.106 recibieron placebo por un promedio de siete años. Se encontró que no hubo diferencia en la incidencia de cáncer colorrectal invasivo entre los dos grupos. Aunque algunos autores han criticado este estudio por la dosis baja de vitamina D utilizada, lo que se espera que aumente la $25-\mathrm{OH}$ vitamina D luego de $400 \mathrm{UI}$ es menos de $3 \mathrm{ng} / \mathrm{ml}$. También, han criticado este estudio pues, según los datos epidemiológicos, se requiere tomar vitamina D, por lo menos, 10 años para lograr el objetivo de disminuir el riesgo de cáncer colorrectal en estadios tempranos y porque el cáncer de colon tiene un periodo de latencia muy largo (10 a 20 años) ${ }^{62}$.

A pesar de la falta de estudios a gran escala, la mayoría de los autores recomienda el suplemento de vitamina D para alcanzar niveles óptimos y evitar el desarrollo de cáncer de colon ${ }^{63}$.

El de próstata es la tercera causa de cáncer en hombres en todo el mundo. Se hizo un metanálisis buscando la relación entre este y la vitamina D. Cuando se evaluó la relación con la vitamina D de la dieta, se encontró que los pacientes que consumían menores cantidades de vitamina $\mathrm{D}$ tenían mayor riesgo de sufrir cáncer de próstata. Se ha demostrado que el consumo de 4.600 UI de vitamina D reduce en $40 \%$ el riesgo de sufrirlo, lo cual indica que la cantidad de vitamina D obtenida por la dieta no es suficiente para prevenir el riesgo de cáncer de próstata. Se han llevado a cabo hasta el momento 10 estudios en los que se evalúa la relación entre la exposición solar y el riesgo de sufrir cáncer de próstata, y en nueve de ellos se encontró un papel protector de la exposición solar. A pesar de que los estudios epidemiológicos son conflictivos, no se debe ignorar el hecho de que la deficiencia de vitamina D se ha encontrado en asociación con cáncer de próstata, ya sea por exposición solar, dieta o alteraciones endógenas. Los ensayos clínicos prospectivos darán luz sobre este tema controversial ${ }^{64}$.

El cáncer de mama es una de las principales neoplasias en las mujeres. Se encuentra regulado por estrógenos y progestágenos, pero también, se ha asociado a otros factores ambientales como son la exposición solar y la dieta. En múltiples estudios se ha evaluado la relación de la exposición solar, la vitamina $\mathrm{D}$ y el cáncer de mama, como por ejemplo, el de Esther, et al., para examinar los posibles efectos protectores de la vitamina D en el cáncer de mama. Analizaron datos del National Cohort Study NHANES I Epidemiologic Follow Up Study. Evaluaron la relación de la exposición solar y la dieta y el suplemento de vitamina D con el subsecuente desarrollo de cáncer. Se incluyeron 5.009 mujeres y encontraron que la exposición solar se asociaba a disminución en el riesgo de cáncer de mama en 20 a $33 \%$. Las pacientes con cáncer de mama tenían menor ingestión de vitamina D por la dieta o suplementos ${ }^{65}$.

En otro estudio de Kupper, et al., se evaluó la asociación entre la dieta o suplemento de vitamina D y la exposición solar, y el riesgo de desarrollar cáncer de mama, en una cohorte de mujeres suizas de edad media. Se incluyeron 41.889 mujeres, las cuales se siguieron por un promedio de 12,9 años. De estas, a 840 se les diagnosticó cáncer invasivo de mama, pero no encontraron ninguna relación entre exposición solar o la ingestión de vitamina $\mathrm{D}$ y el riesgo de cáncer de mama ${ }^{66}$.

Aunque la información sobre la relación entre vitamina D y cáncer de mama está inconclusa, es indiscutible que las células mamarias están bajo la regulación de la vitamina $\mathrm{D}$ y es muy probable que los niveles bajos de vitamina D sí se relacionen con el desarrollo 
de cáncer de mama. Se requieren ensayos clínicos que confirmen estos hallazgos ${ }^{67}$.

El melanoma es el cáncer de piel más letal y se asocia con exposición intermitente intensa al sol y por quemaduras solares en la niñez, pero no con exposición crónica, y para algunos, esta es protectora probablemente debido a la síntesis de vitamina D. Las células de melanoma cultivadas pueden sintetizar calcitriol a partir de la 25-OH vitamina $\mathrm{D}$, expresan los VDR, proliferan más despacio como respuesta al calcitriol; éste induce apoptosis in vitro de las células de melanoma maligno humano, tiene un efecto inhibitorio en las células que diseminan el melanoma, y evita la invasión tumoral y la angiogénesis. La asociación del polimorfismo de VDR con melanoma poco se ha estudiado, pero se ha encontrado que el alelo $f$ del fokI se asocia con un aumento en el riesgo de melanoma y el alelo B en el BsmI se asocio con una disminución en el riesgo de melanoma y se ha visto que el polimorfismo del VDR es más importante en el pronóstico del paciente que en el desarrollo como tal del tumor ${ }^{68}$.

En muchos estudios se ha demostrado que la incidencia del melanoma aumenta con latitudes más cercanas al Ecuador terrestre, lo cual sugiere que el efecto mutagénico de la luz solar es mayor que el efecto protector de la síntesis cutánea de vitamina D. No obstante, en latitudes más altas, menos extremas, con exposición solar limitada, el equilibrio entre protección y daño del sol puede cambiar, incluso, en Europa se ha encontrado una relación reversa con las latitudes. Hay pocos estudios sobre la influencia de las latitudes sobre el melanoma; en un estudio en Australia, se demostró que, a pesar de que vivían más cerca del Ecuador, tenían más incidencia de melanoma, pero las lesiones eran más in situ y menos gruesas. Esto también se demostró en otro estudio; Nürnberg, et al., encontraron que los pacientes que presentaban melanoma en estadio IV tenían niveles más bajos de vitamina $\mathrm{D}$, y aquellos con $25-\mathrm{OH}$ vitamina $\mathrm{D}$ de menos de $10 \mathrm{ng} / \mathrm{ml}$ presentaban los tumores más gruesos y metástasis a distancia más temprano ${ }^{69}$.

La asociación entre radiación solar y melanoma es muy compleja, y se requieren más estudios. Por el momento, se recomienda continuar la protección solar y asegurar niveles adecuados de vitamina D, lo cual puede lograrse con dieta y suplemento ${ }^{70}$.

\section{Enfermedades autoinmunitarias}

La deficiencia de vitamina $\mathrm{D}$ se ha asociado a diabetes mellitus de tipo 1, esclerosis múltiple, artritis reumatoide y lupus eritematoso sistémico, entre otras enfermedades. Se ha observado que la ingestión de $2.000 \mathrm{UI}$ diarias de vitamina $\mathrm{D}$ reduce en $78 \%$ el riesgo de desa- rrollar diabetes mellitus de tipo 1; también, que vivir en latitudes por encima de $35^{\circ}$ en los primeros 10 años de la vida aumenta el riesgo de desarrollar esclerosis múltiple en $100 \%$. Las mujeres que ingieren 400 UI diarias de vitamina D tienen $42 \%$ menos riesgo de sufrir esclerosis y $40 \%$ de artritis reumatoidea y osteoartritis. Se ha demostrado que los pacientes con lupus eritematoso sistémico tienen niveles más bajos de vitamina $\mathrm{D}$ y que esto puede ser un factor de riesgo para el desarrollo de la enfermedad. Por esto, es fundamental realizar tamización y dar suplemento en caso de ser necesario (porque la protección solar es indispensable), ya que en estudios in vitro en ratones lograr niveles adecuados de vitamina $\mathrm{D}$ se asocia con menos proteinuria y mayor supervivencia $^{71}$.

\section{Enfermedad cardiovascular}

En 1997, Rostand reportó que vivir en latitudes altas se asociaba con aumento en el riesgo de sufrir hipertensión arterial sistémica; a esto siguieron otras observaciones según las cuales los pacientes con hipertensión arterial sistémica y con niveles más altos de vitamina D circulante, expuestos a la radiación UVB, tenían niveles más bajos de presión arterial sistólica y diastólica. Existen reportes recientes que afirman que la deficiencia de vitamina $D$ aumenta el riesgo de infarto de miocardio por más del $50 \%{ }^{72}$. En un estudio de una cohorte de 3.358 pacientes, se encontró que 463 (2,8 \%) murieron de causa cardiaca y, $251(34,1 \%)$, de causa no cardiaca, y se demostró que la muerte cardiovascular fue más frecuente con niveles más bajos de vitamina $\mathrm{D}^{73}$. En otro estudio se evaluó prospectivamente la relación del estatus de la vitamina D con la incidencia de eventos cardiovasculares en individuos libres de enfermedad cardiovascular de base. Se incluyeron 1.739 pacientes; de estos, 120 tuvieron un evento cardiovascular primario, la tasa de enfermedad cardiovascular fue dos veces más alta en pacientes con menos de $15 \mathrm{ng} / \mathrm{ml}$ de vitamina D que en aquellos con más de $15 \mathrm{ng} / \mathrm{ml}$. Con estos resultados, pudieron concluir que la deficiencia de vitamina D es un factor de riesgo independiente para el desarrollo de enfermedad cardiovascular. Se requieren más estudios que validen estos datos y determinar si el suplemento de vitamina D contribuirá a la prevención de enfermedades cardiovasculares ${ }^{74}$.

\section{Cómo prevenir la deficiencia de} vitamina D

Para los dermatólogos, la determinación de la $25-\mathrm{OH}$ hidroxivitamina D y el suplemento adecuado con base 
en estos niveles, pueden ser la mejor opción para la prevención del cáncer de piel cuando existen niveles bajos de vitamina $\mathrm{D}$, particularmente, para aquellos pacientes que se encuentran en riesgo de desarrollar deficiencia, como son los niños alimentados con lactancia materna, adultos mayores, o con exposición solar limitada, ya sea por enfermedad, uso de protector solar o costumbre cultural, pacientes de raza negra, obesos o quienes presentan malabsorción. Sin embargo, desde el punto de vista de salud pública, el mejor método para manejar el estatus de la vitamina D es la fortificación de los alimentos. El suplemento se recomienda según la edad: de o a 50 años, debe ser de 200 UI diarias, de 51 a 70 años, de 400 UI diarias, y para mayores de 71 años, de 600 UI diarias. La American Academy of Dermatology, la American Academy of Pediatrics y el Consenso Nacional de da Prevención de Cáncer de Piel, recomiendan que en pacientes en riesgo de deficiencia, la vitamina $\mathrm{D}$ se debe obtener de la dieta o con suplemento, y no por exposición solar; además, recomiendan dosis más altas de vitamina D, así: adultos 1.000 a 2.000 UI diarias, y para niños, 400 UI diarias. Cuando ya existe deficiencia como tal, el suplemento debe ser de 50.000 UI cada semana por ocho semanas 75,76 .

Las recomendaciones de fotoprotección de los dermatólogos deben ser moderadas. Es importante advertir al paciente sobre evitar la exposición solar prolongada, evitar la exposición al medio día en todas las latitudes, usar ropa protectora y buscar la sombra. Las exposiciones muy cortas y limitadas son suficientes para alcanzar niveles adecuados de vitamina D. La exposición del cuerpo en vestido de baño a una dosis de eritema mínimo, es equivalente a ingerir 10.000 UI de vitamina D y se ha reportado que la exposición de $18 \%$ de superficie corporal (manos, cara, brazos) 2 a 3 veces por semana entre un tercio y la mitad de eritema mínimo (aproximadamente, cinco minutos para adultos de fototipo II) en primavera, verano y otoño, es suficiente. Cualquiera que quiera o intente exponerse más de esto, debe protegerse con un protector solar adecuado para prevenir quemaduras y efectos dañinos de exposición solar, ya que la exposición solar prolongada se considera innecesaria y la mayoría de las personas que la práctican no lo hacen por salud sino por lograr un bronceado atractivo ${ }^{77}$.

\section{Referencias}

1. Dusso A, Brown A, Slatopolsky E. Vitamin D. Am J Physiol Renal Physiol. 2005;289:F8-28.

2. Lehmann B, Meurer M. Vitamin D metabolism. Dermatol Ther. 2010;23:2-12.

3. Lips P. Vitamin D physiology. Prog Biophys Mol Biol. 2006;92:4-8.
4. Kochupillai N. The physiology of vitamin D: Current concepts. Indian J Med Res. 2008;127:256-62.

5. Prentice A, Goldberg GR, Schoenmakers I. Vitamin D across the lifecycle: Physiology and biomarkers. Am J Clin Nutr. 2008;88:500S-6.

6. Norman A. From vitamin D to hormone D: Fundamentals of the vitamin D endocrine system essential for good health. Am J Clin Nutr. 2008;88:491-9.

7. Holick M. Vitamin D: A D-Lightful health perspective. Nutr Rev. 2008;66:182-94.

8. Jurutka P, BartikJ, Whitfield G, Mathern D, Barthel T, Gurevich $\mathrm{M}$, et al. D receptor: Key roles in bone mineral pathophysiology, molecular mechanism of action, and novel nutritional ligands. J Bone Miner Res. 2007;22:2-10.

9. Holick M. The cutaneous photosynthesis of previtamin D3: A unique photoendocrine system. J Invest Dermatol. 1981;77:51-8.

10. Bandeira F, Griz L, Dreyer P, Eufrazino C, Bandeira C, Freese E. Vitamin D deficiency: A global perspective. Nutr Rev. 2008;66:S153-64.

11. Bandeira F, Griz L, Dreyer P, Eufrazino C, Bandeira C, Freese E. Vitamin D deficiency: A global perspective. Arq Bras Endocrinol Metabol. 2006;50:640-6.

12. Holick M. Vitamin D deficiency. N Engl J Med. 2007;357:266-81.

13. Holick MF. Sunlight and vitamin $D$ for bone health and prevention of autoimmune diseases, cancers, and cardiovascular disease. Am J Clin Nutr. 2004;80:1678-88.

14. Calvo M, Whiting S, Barton C. Vitamin D intake: A global perspective of current status. J Nutr. 2005;135:310-6.

15. Hanley D, Davison K. Vitamin D insufficiency in North America. J Nutr. 2005;135:332-7.

16. Ashwell M, Stone EM, Stolte H, Cashman KD, Macdonald H, Lanham-New S, et al. Fraser DUK Food Standards Agency Workshop Report: An investigation of the relative contributions of diet and sunlight to vitamin D status. 2010;104:603-11.

17. Vanchinathan V, Lim HW. A dermatologist's perspective on vitamin D. Mayo Clin Proc. 2012 Apr;87(4):372-80.

18. Stechschulte S, Robert B, Kirsner S, Federman D. Vitamin D: Bone and beyond, rationale and recommendations for supplementation. Am J Med. 2009;122:793-802.

19. Cranney A, Horsley T, O’Donnell S, Weiler H, Puil L, Ooi D, et al. Effectiveness and safety of vitamin D in relation to bone health. Evid Rep Technol Assess. 2007;158:1-235.

20. Bacchetta J, Ranchin B, Dubourg L, Cochat P. Vitamin D revisited: A cornerstone of health? Arch Pediatr. 2010;13:115-20.

21. Nagpal S, Rathnachalam R. Noncalcemic actions of vitamin D receptor ligands. Endocr Rev. 2005;26:662-87.

22. DeLuca HF. Overview of general physiologic, features and functions of vitamin D. Am J Clin Nutr. 2004;80:1689-96

23. Lehmann B, Querings K, Reichrath J. Vitamin D and skin: New aspects for dermatology. Exp Dermatol. 2004;3:11-5.

24. Reichrath J, Perez A, Chen TC, Kerber A, Bahmer FA, Holick MF. The effectiveness of topical 1,25-dihydroxyvitamin D3 
$\left(1,25(\mathrm{OH})_{2} \mathrm{D}_{3}\right)$ application in the treatment of psoriasis: An immunohistological evaluation. Acta Derm Venereol. 1997;77:268-72.

25. Bikle D. Vitamin D and the skin. J Bone Miner Metab. 2010;28:117-30.

26. Lehmann B. Role of the vitamin D3 pathway in healthy and diseased skin facts, contradictions and hypotheses. Exp Dermatol. 2009;18:97-108.

27. Banerjee $P$, Chatterjee $M$. Antiproliferative role of vitamin $D$ and its analogs-a brief overview. Mol Cell Biochem. 2003;253:247-54.

28. Walker VP, Modlin RL. The vitamin D connection to pediatric infections and immune function. Pediatr Res. 2009;65:106-13.

29. van Etten E, Decallone B, Verlinden L, Verstuyf A, Bouillon R, Mathieu C. Analogs of 1a,25 dihydroxyvitamin D3 as pluripotent immunomodulators. J Cell Biochem. 2003;88:223-26.

30. Heine G, Anton K, Henz B M, Worm M. 1a,25-D hydroxyvitaminD3 inhibits anti-CD40 plus IL-4-mediated IgE production in vitro. Eur J Immunol. 2002;32:3395-404.

31. Katayama I, Minatohara K, Yokozeki H, NishiokaK. Topical vitamin D3 downregulates IgE-mediated murine biphasic cutaneous reactions. Int Arch Allergy Immunol 1996;111:71-6.

32. Miller J, Gallo R. Vitamin D and innate immunity. Dermatol Ther. 2010;23:12-22.

33. Hong SP, Kim MJ, Jung MY, Jeo H, Goo J, Ahn SK, et al. Biopositive effects of low-dose UVB on epidermis: Coordinate upregulation of antimicrobial peptides and permeability barrier reinforcement. J Invest Dermatol 2008;128:2880-7.

34. Schauber J, Gallo RL. Antimicrobial peptides and the skin immune defense system. J Allergy Clin Immunol. 2008;122:261-6.

35. Fabri M, Modlin RL. A vitamin for autophagy. Cell Host Microbe 2009;6:201-3.

36. Ho-Pham L, Nguyen N, Nguyen T, Bui P, Nguyen V, Nguyen T. Association between vitamin $\mathrm{D}$ insufficiency an tuberculosis in a Vietnamese population. BMC Infect Dis. 2010;25:306-10.

37. Hanada K, Sawamura D, Nakano H, Hashimoto I. Possible role of 1,25-dihydroxyvitamin D3-induced metallothionein in photoprotection against UVB injury in mouse skin and cultured rat keratinocytes. J Dermatol Sci. 1995;9:203-8.

38. Reichrath J. Vitamin D and the skin: An ancient friend, revisited. Exp Dermatol. 2007;16:618-25.

39. Oudshoorn C, Mattace-Raso FU, van der Velde N, Colin EM, van der Cammen TJ, et al. Higher serum vitamin D3 levels are associated with better cognitive test performance in patients with Alzheimer's disease. Dement Geriatr Cogn Disord. 2008;25:539-43.

40. Buell JS, Dawson-Hughes B. Vitamin D and neurocognitive dysfunction: Preventing “D”ecline? Mol Aspects Med. 2008;29:415-22.

41. D’Holick MF, Siris ES, Binkley N. Prevalence of vitamin D inadequacy among postmenopausal North American women receiving osteoporosis therapy. J Clin Endocrinol Metab. 2005;90:3215-24.

42. Mullin GE, Turnbull L, Kines K. Vitamin D: A D-lightful health supplement. Nutr Clin Pract. 2009;24:642-4.

43. Holick M. High prevalence of vitamin D inadequacy and implications for health. Mayo Clin Proc. 2006;81:353-73.

44. Bischoff-Ferrari HA, Giovannucci E, Willett WC, Dietrich T, Dawson-Hughes B. Estimation of optimal serum concentrations of 25-hydroxyvitamin D for multiple health outcomes. Am J Clin
Nutr. 2006;84:18-28.

45. Malabanan A, Veronikis IE, Holick MF. Redefining vitamin D insufficiency. Lancet. 1998;351:805-6.

46. Morten KB, Schmedes B, Philipsen P, Thieden E, Wulf H. Vitamin $D$ production after UVB exposure depends on baseline vitamin $\mathrm{D}$ and total cholesterol but not on skin pigmentation. J Invest Dermatol. 2010;130:546-53.

47. Chen T, Chimeh F, Lua Z, Mathieu J, Person K, Zhang A, et al. Factors that influence the cutaneous synthesis and dietary sources of vitamin D. Arch Biochem Biophys. 2007;460:213-7.

48. Parra E. Human pigmentation variation: Evolution, genetic basis, and implications for public health. Am J Phys Anthropol. 2007;45:85-105

49. Kull M, Kallikorm R, Lember M. Body mass index determines sunbathing habits: Implications on vitamin D levels. Intern Med J. 2009;39:256-8.

50. Wortsman J, Matsuoka J, Chen T, Lu Z, Holick M. Decreased bioavailability of vitamin D in obesity. Am J Clin Nutr. 2000;72:690-3.

51. Alemzadeha R, Kichlerb J, Babara G, Calhouna M. Hypovitaminosis D in obese children and adolescents: Relationship with adiposity, insulin sensitivity, ethnicity, and season. Metabolism. 2008;57:183-91.

52. Holick M. Environmental factors that influence the cutaneous production of vitamin D. Am J Clin Nutr. 1995;61:638S-45S.

53. Matsuoka L, Wortsman J, Hanifan N, Holick M. Chronic sunscreen use decreases circulating concentrations of 5-hydroxyvitamin D. A preliminary study. Arch Dermatol. 1988;124:1802-4.

54. Querings K, Girndt M, Geisel J, George T, Tilgen W, Reichrath J. 25-hydroxyvitamin D deficiency in renal transplant recipients. J Clin Endocrinol Metab. 2006;91:526-9.

55. Marks R, Foley P, Jolley D, Knight K, Harrison J, Thompson S. The effect of regular sunscreen use on vitamin D levels in an Australian population. Results of a randomized controlled trial. Arch Dermatol. 1995;131:415-21.

56. Reichrath J. Skin cancer prevention and UV-protection: How to avoid vitamin D-deficiency? Brit J Dermatol. 2009;161:60.

57. Hatun S, Islam O, Cizmecioglu F, Kara B, Babaoglu K, Berk F, Gökalp AS. Subclinical vitamin D deficiency is increased in adolescent girls who wear concealing clothing. J Nutr. 2005;135:218-2.

58. Grant W. An estimate of premature cancer mortality in the U.S. due to inadequate doses of solar ultraviolet-B radiation. Cancer. 2002;94:1867-75.

59. Holick M. Vitamin D and sunlight: Strategies for cancer prevention and other health benefits. Clin J Am Soc Nephrol. 2008;3:1548-54.

6o. Moan J, Porojnicu A, Robsahm T, Dahlback A, Juzeniene A, Tretli S, Grant W. Solar radiation, vitamin D and survival rate of colon cancer in Norway. J Photochem Photobiol B. 2005;78:189-93.

61. Kampman E, Slattery M, Caan B, Potter J. Calcium, vitamin D, sunshine exposure, dairy products and colon cancer risk (United States). Cancer Causes Control. 2000;11:459-66.

62. Wactawski-Wende J, Kotchen J, Anderson G, Assaf A, Brunner R, O'Sullivan M, et al. Calcium plus vitamin D supplementation and the risk of colorectal cancer. N Engl J Med. 2006;354:684-96.

63. Giovannucci E. Epidemiological evidence for vitamin D and colorectal cancer. J Bone Miner Res. 2007; 22:81-5. 
64. Gupta D, Lammersfeld CA, Trukova K, Lis CG. Vitamin D and prostate cancer risk: A review of the epidemiological literature. Prostate Cancer Prostatic Dis. 2009;12:215-26.

65. John E, Schwartz G, Dreon M, Koo J. Vitamin D and breast cancer risk: The NHANES I epidemiologic follow-up study, 1971-1975 to 1992. Cancer Epidemiol Biomarkers Prev. 1999;8:399-406.

66. Kuper H, Yang L, Sandin S, Lof M, Adami H, Weiderpass E. Prospective study of solar exposure, dietary vitamin $D$ intake, and risk of breast cancer among middle-aged women. Cancer Epidemiol Biomarkers Prev. 2009;18:2558-61.

67. Pérez-López F, Chedraui P, Haya J, Vitamin D acquisition and breast cancer risk. Reprod Sci. 2009;16:7-19.

68. Gandinia S, Raimondia S, Gnagnarellaa P, Dore’b J, Maisonneuvea P, Testoric A. Vitamin D and skin cancer: A meta-analysis. Eur J Cancer. 2009;45:634-41.

69. Nürnberg B, Gräber S, Gärtner B, Geisel J, Pföhler C, Schadendorf D, Tilgen W, Reichrath J. Reduced serum 25-hydroxyvitamin D levels in stage IV melanoma patients. Anticancer Res. 2009;29:3669-74.

70. Osborne J, Hutchinson J. Vitamin D and systemic cancer: Is this relevant to malignant melanoma? Br J Dermatol. 2002;147:197-213.
71. Kamen D, Cooper G, Bouali H, Shaftman S Hollis B, Gilkeson G. Vitamin D deficiency in systemic lupus erythematosus. Autoimmun Rev. 2006;5:114-7.

72. Holick M. The vitamin D deficiency pandemic and consequences for nonskeletal health: Mechanisms of action. Mol Aspects Med.2008;29:361-8.

73. Dobnig H, Pilz S, Scharnagl H, Renner W, Seelhorst U, Wellnitz $\mathrm{B}$, Kinkeldei J, et al. Independent association of low serum 25-hydroxyvitamin $\mathrm{D}$ and 1,25-dihydroxyvitamin D levels with all-cause and aardiovascular mortality. Arch Intern Med. 2008;168:1340-9.

74. Wang T, Pencina $\mathrm{M}$, Booth $\mathrm{S}$, Jacques $\mathrm{P}$, Ingelsson $\mathrm{E}$, Lanier $\mathrm{K}$, et al. Vitamin D deficiency and risk of cardiovascular disease. Circulation. 2008;117:503-11.

75. Weinstock M, Moses A. Skin cancer meets vitamin D: The way forward for dermatology and public health. J Am Acad Dermatol. 2009;61:720-4.

76. Robert J, Lim W. Recommendations on photoprotection and vitamin D. Dermatol Ther. 2010;23:82-5.

77. Reichrath J. Protecting against adverse effects of sun protection. J Am Acad Dermatol. 2003;49:1204-6. 